\title{
Análise Multielementar de solos: uma proposta envolvendo equipamento portátil de fluorescência de raios $\mathrm{X}$
}

\section{Multielement analysis of soils: a proposal involving a portable $X$-ray fluorescence equipment}

\author{
Juliana Terra ${ }^{1}$; Rafael Ortiz Sanches²; Maria Izabel Maretti Silveira Bueno³; Fábio Luiz \\ Melquiades $^{4}$
}

\begin{abstract}
Equipamentos portáteis são escolhas excelentes para a agricultura de precisão, visto que eles permitem obter informações in situ da composição do solo. Neste sentido, os resultados são obtidos rapidamente e implementações podem ser aplicadas imediatamente, dependendo das características do solo local. Este estudo tem o objetivo de desenvolver um método para medida de elementos inorgânicos em solos in natura utilizando um equipamento portátil de EDXRF (Fluorescência de Raios X por Dispersão em Energia) e calibração multivariada por regressão PLS (Mínimos Quadrados Parciais). Sete materiais de referência certificados foram utilizados para construir os modelos PLS para Si, K, Ca, Mn e Fe. Dois padrões foram utilizados para a validação externa do modelo. Os resultados mostram que o desvio relativo máximo obtido foi de $21 \%$.

Palavras-chave: Solo. Fluorescência de raios X. Agricultura de precisão.
\end{abstract}

\section{Resumo}

Portable instruments are excellent choices to perform precision agriculture since they allow in situ information about soil composition. In such a way, results are obtained quickly and inputs can be applied promptly, depending on the local soil characteristics. This study aims to develop a method to measure inorganic elements at in natura soils using a portable EDXRF (Energy Dispersive X-ray Fluorescence) equipment and multivariate calibration through Partial Least Square Regression (PLS). Seven certified reference standards are used to build the PLS models for $\mathrm{Si}, \mathrm{K}, \mathrm{Ca}, \mathrm{Mn}$ and $\mathrm{Fe}$. Two standards are separated for external PLS validation. Finally, the results show that a maximum of $21 \%$ is obtained for relative deviation.

Key words: Soil. X-ray fluorescence. Precision farming.

\footnotetext{
${ }^{1}$ Graduada e Pós-Doc em Química, Universidade Estadual de Campinas; jut@uol.com.br

${ }^{2}$ Aluno de graduação em química, Universidade Estadual de Campinas; rafael.ortiz.sanchez@gmail.com

${ }^{3}$ Docente do Instituto de Química, Universidade Estadual de Campinas; bell@iqm.unicamp.br

${ }^{4}$ Docente do Departamento de Física, Universidade Estadual do Centro-Oeste; fmelquiades@unicentro.br
} 


\section{Introdução}

O estudo sobre as características do solo é fundamental para se obter boa produtividade na agricultura. Quando o solo não dispõe de nutrientes suficientes, normalmente são utilizadas técnicas de fertilização artificiais. A quantidade e o tipo de fertilizante necessário variam segundo o cultivo, a época e as características do solo. A fertilização inadequada provoca um desequilíbrio dos nutrientes essenciais através da falta ou excesso destes (principalmente nitrogênio, fósforo, potássio e cálcio), e tem por consequência um aumento no custo de produção do cultivo (MAULE; MAZZA; MARTHA JÚNIOR, 2001), (LEMOS; NOGUEIRA; TORRE NETO, 2004).

Segundo Fageria (1998), o custo da aplicação de fertilizantes contribui, em média, com $30 \%$ do custo total da produção em culturas anuais, o que permite afirmar que um estudo prévio da composição nutricional do solo é fundamental, a fim de otimizar o teor e disponibilidade dos elementos essenciais para melhorar a produtividade e reduzir o custo de produção.

De acordo com Coghlan (1995) e Mapa e Kumaragamage (1996), a agricultura de precisão pode ser definida como um conjunto de tecnologias que visam gerenciar a produção, maximizando a rentabilidade das colheitas e minimizando o impacto ambiental, e que leve em consideração a variabilidade espacial e temporal das culturas, do solo e do clima. Portanto, o mapeamento prévio da composição nutricional do solo está diretamente ligado à agricultura de precisão.

Equipamentos portáteis que permitem obter informações do solo in situ apresentam vantagens frente aos de bancada, uma vez que aceleram a obtenção do resultado e permitem alocar os insumos com base nas necessidades de cada área no campo. Técnicas envolvendo tais equipamentos, se associadas a outras características como baixo custo e respeito à Química Verde, são ainda mais convenientes de serem exploradas (TERRA et al. 2010a).

Dentre estas técnicas, é possível citar a fluorescência de raios $\mathrm{X}$ (FRX), cuja real potencialidade para análise de solo e sedimento com equipamentos portáteis foi avaliada e comprovada anteriormente (INTERNATIONAL ATOMIC ENERGY AGENCY, 2005), (UNITED STATES ENVIRONMENTAL PROTECTION AGENCY, 2006), e já é uma metodologia estabelecida e em crescente uso em estudos ambientais.

O princípio da FRX está relacionado com a medida de energia e intensidade características da radiação $\mathrm{X}$ emitida por uma amostra irradiada com radiação eletromagnética de alta energia. As principais vantagens de equipamentos portáteis de FRX incluem resultados imediatos em campo, análise não destrutiva, determinação simultânea de diversos elementos, velocidade de operação.

Segundo a International Union of Pure and Applied Chemistry (2012), a radiação X pode ser definida como a radiação resultante da interação de partículas de alta energia ou fótons com a matéria. O princípio da FRX pode ser descrito quando uma partícula ou um fóton com alta energia atinge um elétron das camadas internas de um átomo, e a energia (E) dessa partícula é maior que a energia de ligação (E0) do elétron no átomo, permitindo que o elétron seja ejetado, com energia cinética (E - E0), ou seja, equivalente à diferença entre a energia inicial da partícula e a energia de ligação do elétron no átomo. O elétron ejetado é chamado fotoelétron e esse fenômeno é denominado efeito fotoelétrico. Após o efeito fotoelétrico, há um rearranjo eletrônico, com emissões de raios $\mathrm{X}$ características. Como cada elemento libera determinadas energias neste processo, podem-se obter informações qualitativas da amostra. Paralelamente, a intensidade da energia emitida é proporcional à quantidade do elemento na amostra (JENKINS, 1999).

A fluorescência de raios $\mathrm{X}$, quando aliada à quimiometria, possibilita extrair maior informação dos dados multivariados (TERRA et al., 2010b), 
(BODE et al., 2008), (CALZA et al., 2007). Entre os métodos quimiométricos quantitativos, a regressão por mínimos quadrados parciais, PLS (do inglês Partial Least Square) tem sido frequentemente utilizada nos mais diversos campos da ciência.

A regressão PLS estabelece uma relação quantitativa entre o conjunto das respostas instrumentais (por exemplo, dados espectrais) e uma ou mais propriedades físicas ou químicas das amostras de interesse (concentração, acidez, viscosidade, etc.), desenvolvendo um modelo matemático que correlaciona estas informações. Este procedimento engloba duas etapas: calibração e validação. A etapa de calibração estabelece uma relação entre a matriz inicial de dados X (sinais instrumentais, variáveis independentes) e as propriedades conhecidas de amostras de referência (variáveis dependentes) organizados na matriz Y. A validação permite verificar se o modelo é capaz de prever as propriedades de novas amostras - valores de incógnitas (TERRA, 2009), (OTTO, 1999).

O objetivo deste trabalho foi elaborar um método para quantificar simultaneamente $\mathrm{Si}, \mathrm{K}, \mathrm{Ca}, \mathrm{Mn}$ e Fe em solo in natura, fazendo uso de equipamento portátil de fluorescência de raios X com dispersão de energia e calibração multivariada por PLS.

\section{Materiais e Métodos}

Foram utilizados 7 materiais de referência certificados, cuja descrição e valores de concentração para os elementos avaliados encontram-se na Tabela 1. A granulometria especificada em todos os certificados dos padrões era $<70 \mu \mathrm{m}$.

Para a obtenção das medidas por FRX, foram utilizadas celas apropriadas (Chemplex 1340) sustentadas por filme de Mylar ${ }^{\circledR}$ (Chemplex 100) com 2,5 $\mu \mathrm{m}$ de espessura. Visto que os materiais de referência estavam com granulometria e teor de umidade adequados, as amostras foram acondicionadas diretamente nos recipientes. Foram preparadas duas celas para cada material e estas irradiadas em triplicata, totalizando 42 espectros.

O espectrômetro portátil de fluorescência de raios $\mathrm{X}$ por dispersão de energia (EDXRF) utilizado foi o da marca InnovX, modelo AlphaCam, constituído por um tubo de raios $\mathrm{X}$ com ânodo de tântalo e um detector de diodo de SiPIN.

Durante as irradiações das amostras, a voltagem aplicada no tubo de raios X foi de $40 \mathrm{kV}$ e a corrente igual a $7 \mu \mathrm{A}$. Foi utilizado filtro de alumínio de 100 $\mu \mathrm{m}$ entre o feixe do tubo e a amostra e o tempo de irradiação foi de $60 \mathrm{~s}$.

\section{Regressão por Mínimos Quadrados Parciais}

Nas construções dos modelos PLS, combinações das variáveis originais da matriz X são realizadas e novas variáveis são geradas, chamadas de variáveis latentes. A escolha do número de variáveis latentes (LV) é uma etapa importante, sendo o valor ideal aquele que permite a construção de um modelo com boa capacidade de previsão para amostras externas, sem super ajustar o modelo e nem promover a modelagem de ruído (MARTENS; NAES, 1996). Os números de LV utilizados neste estudo estão na Tabela 2.

Uma matriz de $(30$ x 2048) referente a 30 espectros (amostras) e 2048 canais de energia do multicanal (variáveis), constituiu a matriz $\mathrm{X}$ de calibração. A matriz Y de calibração foi composta pelos valores de concentração para cada um dos 5 elementos de interesse constituindo uma matriz (30 x 5).

As amostras 2 (SRM 2709) e 5 (NRCC-MESS-2), total de 12 espectros (dois padrões em duplicata medidos 3 vezes cada, ou seja, 4 recipientes vezes 3 medidas), foram selecionadas aleatoriamente para serem as amostras externas e, portanto, não foram utilizadas nas construções dos modelos PLS (calibração e validação interna). As matrizes de validação externa $\mathrm{X}$ e $\mathrm{Y}$ foram compostas de $(12 \mathrm{x}$ 2048) e (12 x 5), respectivamente. 
Os dados espectrais foram centrados na do conjunto de dados fique na origem do sistema de média, procedimento este, que permite facilitar a eixos das componentes principais (OTTO, 1999). visualização dos dados, ao fazer com que o centro

Tabela 1 - Materiais de referência certificados e suas respectivas concentrações em mg kg-1.

\begin{tabular}{cccccccc}
\hline Amostra & Padrão & Descrição & S1 & K & Ca & Fe & Mn \\
\hline 1 & SRM 1646 & Estuarine sediment & 40 & 0,864 & 0,519 & 2,008 & 0,02345 \\
2 & SRM 2709 & San Joaquim soil & 29,66 & 2,03 & 1,89 & 3,5 & 0,0538 \\
3 & SRM 2710 & Montana soil & 28,97 & 2,11 & 1,25 & 3,38 & 1,01 \\
4 & SRM 2711 & Montana soil & 30,44 & 2,45 & 2,88 & 2,89 & 0,0638 \\
5 & NRCC-MESS-2 & Marine sediment & 27,8 & 0 & 0 & 4,3503 & 0,0365 \\
6 & NRCC-PACS-2 & Marine sediment & 27,579 & 1,237 & 1,9654 & 4,0916 & 0 \\
7 & CANMET-SO-2 & Soll & 24,99 & 2,45 & 1,96 & 5,56 & 0,0720 \\
\hline
\end{tabular}

Fonte: Autores

Para validação interna, foi utilizada a validação cruzada, na qual uma amostra é retirada do conjunto de $\mathrm{n}$ amostras e então é feita a calibração para as n-1 amostras restantes (variante "leave-one-out"). Na sequência, a amostra não usada na calibração tem sua variável prevista pelo modelo. O processo é repetido até que todas as amostras tenham sido testadas (BEEBE; PELL; SEASHOLTZ, 1998).

Para a construção dos modelos PLS, foi utilizado o programa computacional Pirouette ${ }^{\circledR} \quad 3.11$ (Infometrix Co., 2003), levando em consideração os espectros médios das replicatas de cada material de referência.

\section{Resultados}

Os espectros de fluorescência de raios $\mathrm{X}$ dos materiais de referência estão apresentados na Figura 1.
Figura 1 - Espectros sobrepostos dos materiais de referência, com detalhe para identificação de picos.

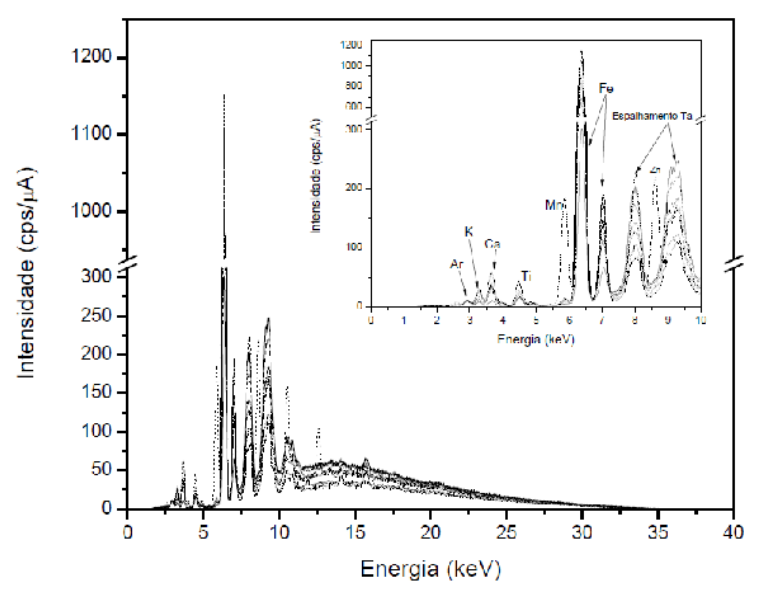

Fonte: Autores

Foram obtidos modelos para todos os elementos analisados, considerando nesta etapa apenas os respectivos picos, o que justifica que tenham sido utilizados números diferentes de variáveis latentes para cada modelo. Os canais de energia, os números de variáveis latentes e as respectivas porcentagens da variância das amostras estão apresentados na Tabela 2. 
Tabela 2 - Faixa de energia e número de variáveis latentes (LV) utilizadas na construção de cada um dos modelos PLS e as respectivas porcentagens de variância explicada.

\begin{tabular}{cccc}
\hline Modelo & $\begin{array}{c}\text { Intervalo de } \\
\text { energia (keV) }\end{array}$ & LV & $\begin{array}{c}\text { Variância } \\
(\%)\end{array}$ \\
\hline $\mathrm{Ca}$ & $3,46-3,90$ & 2 & 99,00 \\
$\mathrm{Fe}$ & $6,10-6,74$ & 3 & 99,96 \\
$\mathrm{~K}$ & $3,01-3,45$ & 1 & 97,53 \\
$\mathrm{Si}$ & $7,39-12,01$ & 4 & 99,81 \\
$\mathrm{Mn}$ & $5,63-6,04$ & 3 & 99,99 \\
\hline
\end{tabular}

Fonte: Autores

As relações entre os valores previstos e os valores de referência estão apresentadas na Figura 2, para o modelo de validação interna de todos os elementos, juntamente com os valores dos coeficientes de correlação (rval) e as equações matemáticas que expressam os modelos. Os valores obtidos da validação interna foram comparados com os valores das concentrações dos metais fornecidos na certificação, permitindo averiguar a exatidão do método, uma vez que o maior erro encontrado para estas amostras foi igual a $11,6 \%$, conforme a Tabela 3.

Figura 2 - Relação entre os valores de previsão (é o como está nos eixos dos gráficos) e os valores de referência obtidos com os modelos PLS para Si, K, $\mathrm{Ca}, \mathrm{Mn}$ e Fe

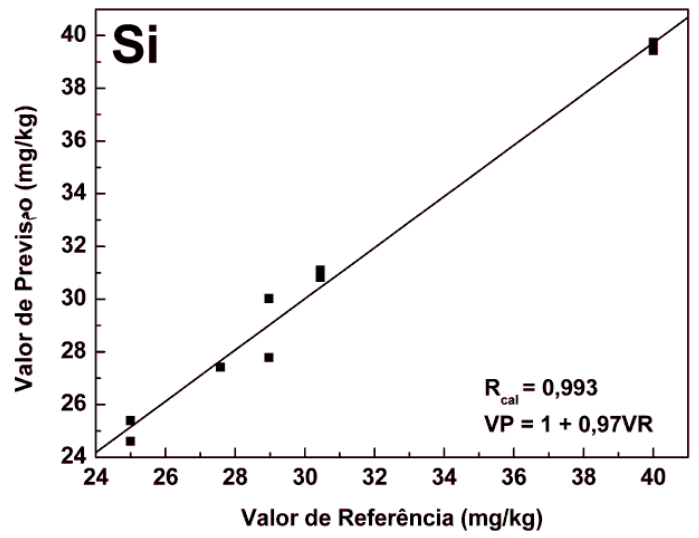

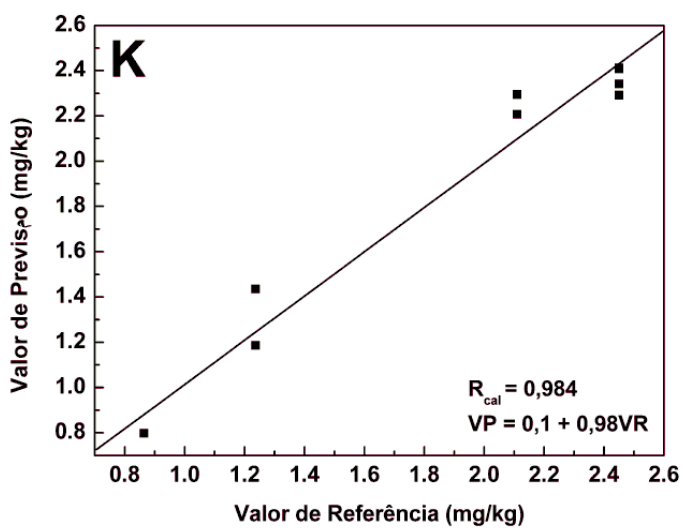
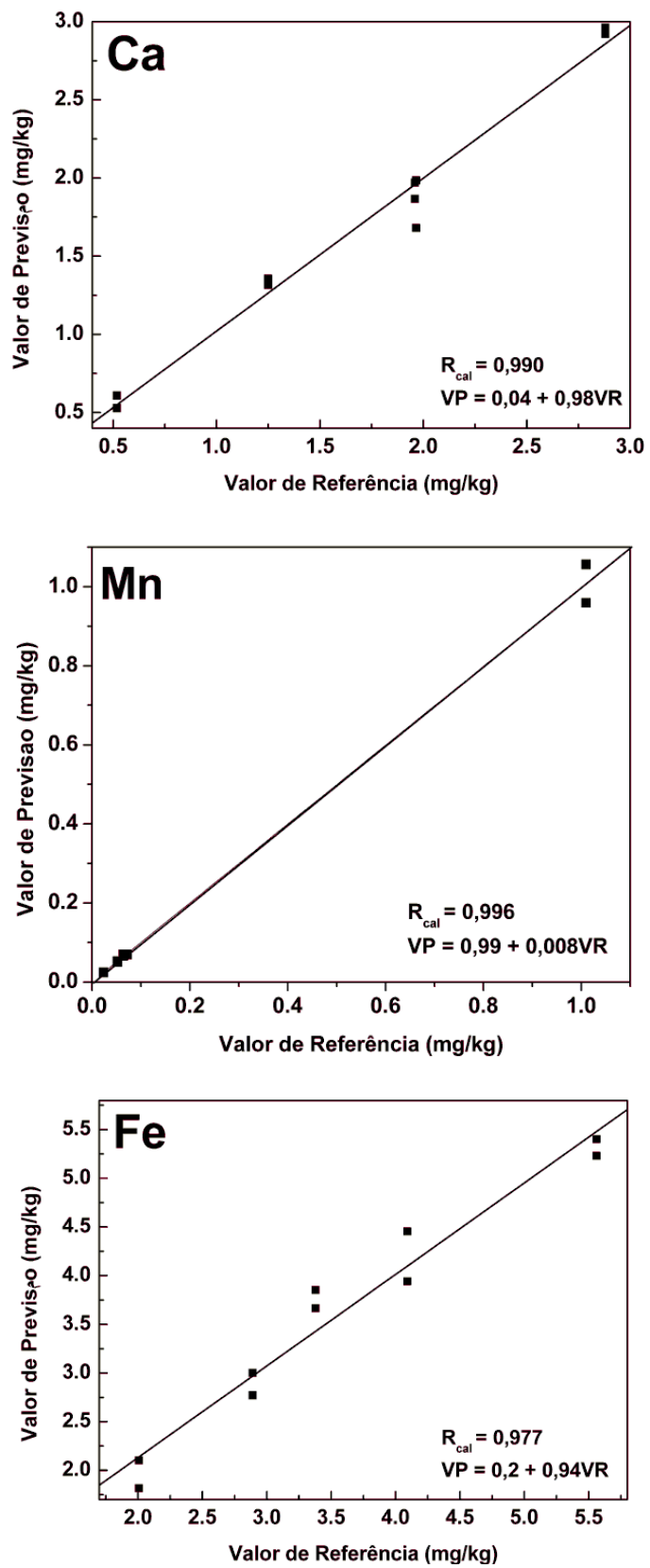

Fonte: Autores 
Tabela 3 - Erros percentuais, em módulo, entre os valores de referência e a média dos valores previstos na validação interna.

\begin{tabular}{cccccc}
\hline Amostra & $\mathbf{S i}$ & $\mathbf{K}$ & $\mathbf{C a}$ & $\mathbf{F e}$ & $\mathbf{M n}$ \\
\hline 1 & 1,0 & 7,7 & 9,5 & 2,6 & 8,7 \\
3 & 0,2 & 6,7 & 6,9 & 11,2 & 0,2 \\
4 & 1,7 & 4,1 & 2,1 & 0,1 & 11,6 \\
6 & 0,6 & 5,9 & 6,8 & 2,6 & $\mathrm{ND}^{*}$ \\
7 & 0,1 & 3,0 & 2,1 & 4,4 & 1,8 \\
\hline
\end{tabular}

ND* valor não declarado na certificação

Fonte: Autores

Posteriormente à construção dos modelos, previsões dos teores dos elementos das amostras externas (materiais de referência 2 e 5) foram realizadas e a comparação com os valores de referência permitiu verificar a potencialidade do método proposto com maior confiabilidade conforme valores da Tabela 4. É possível verificar nesta tabela que o elemento que apresentou maior erro foi o potássio (amostra 2).

Este estudo foi realizado em condições otimizadas quanto a preparação das amostras que, sendo materiais de referência certificadas, são homogêneas quanto à granulometria e umidade. Para aplicação em amostras de solo em campo, correções para estes dois fatores devem ser consideradas, o que não inviabiliza sua utilização (BASTOS; MELQUIADES; BIASI, 2012), (MELQUIADES et al., 2011).

\section{Conclusão}

Foi desenvolvida uma metodologia para a quantificação de $\mathrm{Si}, \mathrm{K}, \mathrm{Ca}, \mathrm{Mn}$ e Fe em amostras de solo in natura com equipamento portátil de EDXRF, cujo desvio relativo foi inferior a $21 \%$. Este valor pode ser considerado satisfatório, em função do uso de dados espectrais, de equipamentos portáteis, da facilidade de medida e rapidez para obtenção dos resultados. Melhores resultados poderiam ser obtidos se matrizes de amostras semelhantes fossem utilizadas para calibração. Contudo, mesmo com o uso de amostras com matrizes diferentes e de diversas origens (solo, sedimentos marinhos, sedimentos de lago), resultados satisfatórios foram obtidos comparativamente às técnicas tradicionais de medida.

Tais resultados permitem verificar a potencialidade da aplicação da técnica de fluorescência de raios $\mathrm{X}$ aliada à quimiometria para se determinar de maneira simultânea e rápida, teores de diversos elementos essenciais em solo.

Tabela 4 - Valores de referência (VR) e médias previstas pelo modelo proposto (VP) para os teores dos elementos em $\mathrm{mg} \mathrm{kg}^{-1}$ e erro percentual em módulo $(|\mathrm{E} \%|)$ entre estes valores na validação externa.

\begin{tabular}{cccc}
\hline & \multicolumn{3}{c}{ Amostra 2 } \\
Elemento & VR & VP & $|\mathbf{E} \%|$ \\
$\mathrm{Si}$ & 29,66 & 34,28 & 16 \\
$\mathrm{~K}$ & 2,03 & 1,61 & 21 \\
$\mathrm{Ca}$ & 1,89 & 1,94 & 3 \\
$\mathrm{Fe}$ & 3,5 & 3,8 & 9 \\
$\mathrm{Mn}$ & 0,0538 & 0,0635 & 8 \\
\hline & & & \\
\hline & & Amostra 5 & \\
Elemento & VR & $\mathbf{V P}$ & $|\mathbf{E} \%|$ \\
$\mathrm{Si}$ & 27,80 & 26,21 & 6 \\
$\mathrm{~K}$ & $\mathrm{ND} *$ & - & - \\
$\mathrm{Ca}$ & $\mathrm{ND} *$ & - & - \\
$\mathrm{Fe}$ & 4,35 & 4,83 & 11 \\
$\mathrm{Mn}$ & 0,0365 & 0,0395 & 18 \\
\hline ND* valores não declarados na certificação
\end{tabular}

Fonte: Autores 


\section{Referências}

BASTOS, R. O.; MELQUIADES, F. L.; BIASI, G. E. V. Correction for the effect of soil moisture on in situ XRF analysis using low-energy background. X-Ray Spectrometry, London, v. 41, p. 304-307, 2012.

BEEBE, K. R.; PELL, R. J.; SEASHOLTZ, M. B. Chemometrics: a practical guide. New York: John Wiley \& Sons, 1998.

BODE, P.; BUENO, M. I. M. S.; BORTOLETO, G. G.; HOFFMANN, G.; VAN DEN INGH, T. S. G. A. M.; ROTHUIZEN. Neutron activation analysis and $\mathrm{X}$-ray Rayleigh and Raman scattering of hair and nail clippings as noninvasive bioindicators for $\mathrm{Cu}$ liver status in labrador retrievers. J. Analytical and Bioanalytical Chemistry, Heidelberg, v. 390, n. 6, p. 1653-1658, 2008.

CALZA, C.; ANJOS, M. J.; BUENO, M. I. M. S.; SOUZA, S. M.; BRANCAGLION JR, A.; LIMA, T. A.; LOPES, R. T. XRF applications in archaeometry: analysis of Marajoara pubic covers and pigments from the sarcophagus cartonage of an Egyptian mummy. X-Ray Spectrometry, London, v. 36, p. 348-354, 2007.

COGHLAN, A. Hi-tech farming to save the environment? New Scientist, London, n. 23, p. 25, 1995.

FAGERIA, N. K. Otimização da eficiência nutricional na produção das culturas. Revista Brasileira de Engenharia Agrícola e Ambiental, Campina Grande, PB, v. 2, n. 1, p. 6-16, 1998.

INTERNATIONAL ATOMIC ENERGY AGENCY - IAEA. In-situ applications of X-ray fluorescence techniques: final report of a coordinated research project 2000-2003 (IAEA-TECDOC-1456). Vienna: International Atomic Energy Agency, 2005. INTERNATIONAL UNION OF PURE AND APPLIED CHEMISTRY IUPAC. IUPAC Compendium of chemical terminology. 2th ed. Disponível em: http://old.iupac.org/goldbook/ X06703.pdf. Acesso em: 24 set. 2013.
JENKINS, R. X-Ray spectrometry. New York: Wiley-Interscience, 1999.

LEMOS, S. G.; NOGUEIRA, A. R. A., TORRE NETO, A. A agricultura na era da informação: desafios e propostas para automação em análises de solos. Revista Analytica, Rio de Janeiro, n. 8, p. 4250, 2004.

MAPA, R. B.; KUMARAGAMAGE, D. Variability of soil properties in a tropical Alfisol used for shifting cultivation. Soil Technology, Amsterdam, v. 9, n. 3, p. 187-197, 2006.

MARTENS, H.; NAES, T. Multivariate calibration. New York: Wiley, 1996.

MAULE, R. F.; MAZZA, J. A.; MARTHA JÚNIOR, G. B. Produtividade agrícola de cultivares de canade-açúcar em diferentes solos e épocas de colheita. Scientia Agrícola, Piracicaba, v. 58, n. 2, p. 295301, 2001.

MELQUIADES, F. L.; BASTOS, R. O.; BIASI, G. E. V.; PARREIRA P. S.; APPOLONI, C. R. Granulometry and moisture influence for in situ soil analysis by portable EDXRF. AIP Conference Proceedings, v. 1351, p. 317-320, 2011.

OTTO, M. Chemometrics: statistics and computer application in analytical chemistry. Weinheim: Wiley, 1999.

TERRA, J. Potencialidade da aliança da espectroscopia de raios $x$ e quimiometria na determinação de valor energético e teores de alguns macronutrientes em amostras de farinhas para consumo humano. 2009. Tese (Doutorado em Química) - Universidade Estadual de Campinas, Campinas.

TERRA, J.; ANTUNES, A. M.; BUENO, M. I. M. S.; PRADO, M. A. Energy value determinations of industrialized foods: the potential of using X-ray spectroscopy and partial least squares. X-Ray Spectrometry, London, v. 39, p. 167-175, 2010 b. 
TERRA, J.; ANTUNES, A. M.; BUENO, M. I. M. S.; PRADO, M. A. Um método verde, rápido e simples para determinar o valor energético de farinhas e cereais matinais. Química Nova, São Paulo, v. 33, n. 5, p. 1098-1103, 2010a.

\section{UNITED STATES ENVIRONMENTAL} PROTECTION AGENCY - USEPA. Innovative Technology Verification Report. EPA/540-R-06/008. Washington: United States Environmental Protection Agency, 2006.

Recebido em 25 Setembro, 2013 - Received on September 25, 2013

Aceito em 20 Julho, 2014 - Accepted on July 20, 2014 